\title{
1 Type I-E CRISPR-Cas system as an immune system in a eukaryote
}

2

3 Devashish Rath ${ }^{1,2^{*}}$, Lina Amlinger ${ }^{2}$, Gargi Bindal ${ }^{1}$, Magnus Lundgren ${ }^{2} \#$

4

$5 \quad{ }^{1}$ Molecular Biology Division, Bhabha Atomic Research Centre, Mumbai, India ${ }^{a}$

$6{ }^{2}$ Department of Cell and Molecular Biology, Uppsala University, Uppsala, Sweden ${ }^{\mathrm{b}}$

7

8 \#Address correspondence to Magnus Lundgren, magnus.lundgren@icm.uu.se

9 *Present address: Molecular Biology Division, Bhabha Atomic Research Centre,

10 Mumbai, India.

11 


\section{Abstract}

Defense against viruses and other mobile genetic elements (MGEs) is important in many organisms. The CRISPR-Cas systems found in bacteria and archaea constitute adaptive immune systems that acquire the ability to recognize MGEs by introducing nucleic acid samples, spacers, in the CRISPR locus. The CRISPR is transcribed and processed, and the produced CRISPR RNAs guide Cas proteins to degrade matching nucleic acid sequences. No CRISPR-Cas system is found to occur naturally in eukaryotic cells but here we demonstrate interference by type I-E CRISPR-Cas system from Escherichia coli introduced in Saccharomyces cerevisiae. The designed CRISPR arrays are properly expressed and processed in S. cerevisiae. Targeted plasmids display reduced transformation efficiency, indicative of DNA cleavage. Unlike e.g. Cas9-based systems, which can be used to inactivate MGEs in eukaryotes by introducing specific mutations, type I-E systems processively degrade the target. The type I-E system thus allows for defense without knowledge of MGE gene function. The reconstituted CRISPR-Cas system in S. cerevisiae can also function as a basic research platform for testing the role of various factors in the interference process.

\section{Introduction}

Viruses and other mobile genetic elements (MGEs) are potential threats to most studied cellular organisms, by acting as predators or by reducing fitness. In response, organisms have evolved multiple defense strategies, largely grouped into innate and adaptive systems. Innate systems are characterized by being activated by certain preset features of infection. Adaptive systems, on the other hand, can learn to recognize previously unrecognized pathogens. For a long time the vertebrate adaptive 
immune system was the only known example, but the CRISPR-Cas systems of archaea and bacteria have been demonstrated to be a bona fide adaptive immune systems (1). All studied CRISPR-Cas systems are based on short DNA or RNA sequences (protospacers) from e.g. virus genomes being stored as DNA spacers in the Clustered Regularly Interspaced Short Palindromic Repeats (CRISPR) locus. A long precursor CRISPR transcript (pre-crRNA) is processed into CRISPR RNA (crRNA) and used by CRISPR associated (Cas) protein effectors to locate and destroy matching targets. The target can be DNA or RNA depending on the type of CRISPR-Cas system (2, 3). CRISPR-Cas systems are grouped into class 1 and 2, depending on if the effector is a protein complex or a single enzyme, respectively. Each class contains several functionally different types of systems (4).

Programmable nucleases such as Zinc finger nucleases (ZFNs), transcription activator like effector nucleases (TALENs), and Cas9 can function as anti-MGE systems in eukaryotic cells by inducing crippling mutations. Particularly Cas9, which has revolutionized gene editing in eukaryotes, has been demonstrated to effectively target several human viruses (5). In basic Cas9 technology DNA cleavage is directed by a single guide RNA (sgRNA). The break can be repaired by Homology Directed Repair (HDR) or error-prone Non-Homologous End Joining (NHEJ) (2). In the case of viruses, if a gene is repaired by the error-prone NHEJ it may be inactivated, resulting in inability of the virus to proliferate, as demonstrated for $e . g$. HIV1 (6). However, this approach requires thorough understanding of the virus' biology, as randomly targeting a virus gene does not guarantee reduced proliferation. 
61 In this study we take advantage of the properties of the type I-E CRISPR-Cas system to test its ability to function as an anti-MGE system in eukaryotes that do not require

63 understanding of target gene function. The key factors in target degradation by type I-

64 E systems are the Cascade protein-RNA complex and the Cas3 enzyme. Cascade

65 processes and retains crRNA, and uses the crRNA to identify DNA targets flanked by

66 a protospacer adjacent motif (PAM) (7). Once a target is identified, the Cas3 enzyme

67 is recruited to degrade the target. Unlike the DNA-targeting class 2 enzymes, which

68 perform blunt or staggered double-stranded cut, Cas3 destroys the target in a

69 processive manner (8). This property makes Cascade-Cas3 a poor choice for gene

70 editing but highly suitable for removing virus genomes as repair that restore virus

71 function is less likely to occur after processive degradation. As the effect should be

72 the same irrespective of target sequence, Cascade-Cas3 would be especially

73 advantageous for use against poorly characterized viruses. A type I-E system in a

74 eukaryote could also be beneficial for basic research. It allows testing the role and

75 effect of Cas and non-Cas proteins, as well as other factors, for their effect on

76 CRISPR-Cas immunity, independent of their native cellular background.

77

78 As a proof-of-concept we adapted the type I-E system from E. coli for use as a

79 programmable anti-MGE system in S. cerevisiae, chosen for its role as a eukaryotic

80 model system. As targets we use plasmids, which allow comparison with similar

81 experiments in bacterial systems (9-12).

82

83 Results

84 Design and reconstitution of type I-E CRISPR-Cas system in S. cerevisiae 
85 Our basic system for expressing Cascade, Cas3, and crRNA in S. cerevisiae was different plasmids from the pRSGal series, where each produced one of the components (fig. 1A), but we also designed several alternative systems (fig. 1B-D). pCascade, expressing Cascade proteins, had three cassettes with different cas genes under control of bidirectional Gal promoters. The cassettes were separated by $S$. cerevisiae $\mathrm{CYC} 1$ terminator to prevent formation of antisense transcripts.

91 For production of targeting crRNA, pCRISPR was constructed by inserting a CRISPR array containing four copies of the $\mathrm{J} 3$ spacer $(4 \times \mathrm{J} 3)$ (13) under control of a Gal promoter, with a CYC1 terminator preventing read-through. a Gal promoter. An alternative version of the plasmid, pCas3::Cse1, carried a naturally occurring cas3-cse1 fusion (8). Co-expression of this plasmid with pCascade would result in incorporation of the Cas3-Cse1 fusion in a part of the Cascade complexes. To test the effect of different crRNAs we combined a CRISPR with two alternative spacers, $\mathrm{J} 1$ and $\mathrm{J} 2$, with Cas3 or Cas3-Cse1 fusion to generate pCRISPR+Cas3 and pCRISPR+Cas3::Cse1, respectively. Two different target plasmids were designed. pTargetHigh carried a phage Lambda J gene fragment containing the PAMs and protospacers for J1, J2, and J3 (table S4) on

103 a high copy vector. pTargetLow, carried the same $\mathbf{J}$ fragment on a low copy

104 backbone. Empty vectors lacking the $\mathrm{J}$ fragment were used as non-targeted controls.

\section{Processing of crRNA in S. cerevisiae}

107 To test function of crRNA processing in yeast we analyzed the formation of crRNA in

108 S. cerevisiae W303 after expression from pCascade, pCas3, and pCRISPR was

109 induced by addition of galactose. The analysis was performed by northern blot with a 
110 radioactively labeled probe complementary to the $\mathrm{J} 3$ spacer. The assay demonstrates

111 formation of a 61 nt RNA species, equal in size to crRNA produced in Escherichia

112 coli. The amount of crRNA produced was assayed two, four, and five hours after

113 induction, and crRNA was detected at all time points (fig. 2A).

115 Interference by CRISPR-Cas in S. cerevisiae

116 Functionality of Cascade-Cas3 interference was assessed by comparing

117 transformation efficiency (TE) of a plasmid targeted by the CRISPR-Cas system with 118 that of the same plasmid lacking the target fragment. Transformations were routinely

119 done 240 min after induction of cas and CRISPR expression. Using pCas3, pCascade, 120 and pCRISPR in S. cerevisiae W303, a 47\% decrease in TE of pTargetHigh was 121 observed (fig. 2B). Tests with $150 \mathrm{~min}$ induction resulted in similar results (fig. S1).

122 Alternative systems for expressing Cascade, Cas3, and crRNA were tested for 123 improved target interference. Cas3-Cse1 fusion construct, pCas3::Cse1, co-expressed 124 with pCascade and pCRISPR resulted in approximately $73.5 \%$ reduction in TE of 125 pTargetHigh in S. cerevisiae W303 (fig. 2C). Expression of pCRISPR+Cas3 or 126 pCRISPR+Cas3::Cse1 together with pCascade in S. cerevisiae BY418 resulted in $12788.6 \%$ and $88.7 \%$ reduction in TE of pTargetLow, respectively (fig. 2D and 2E).

129 Effect of interference

130 Interference assays demonstrated that CRISPR-Cas targeting reduced TE but 131 transformants were still observed on selective plates. These transformants could be 132 carrying target plasmids that had not been cleaved by Cascade-Cas3, plasmids that 133 had been cleaved but subsequently been repaired or plasmids that had acquired escape 134 mutations in the region targeted by CRISPR. NHEJ repair or presence of different 
escape mutations would result in target sequence heterogeneity in the population of transformed plasmids. To determine sequence heterogeneity in the transformed

137 plasmids we performed the Surveyor assay, which cleave DNA with mismatched

138 bases formed by denaturing and reannealing DNA strands with sequence differences.

139 The target region of pTargetHigh was amplified by PCR and analyzed by Surveyor

140 assay. Sequence heterogeneity at J3 target site would result in two cleavage

141 fragments, both of about 160-170 bp. No such cleavage products could be detected in

142 strains expressing pCRISPR, pCascade and pCas3, similar to interference-negative

143 control strains (fig. S2).

\section{Discussion}

146 We demonstrate successful reconstitution of bacterial type I-E CRISPR-Cas

147 interference in the eukaryote $S$. cerevisiae. We detect accurate processing of crRNA

148 by northern blot and, importantly, a reduction of target plasmid transformation rate

149 compared to a non-targeted control plasmid. We could not detect sequence

150 heterogeneity in plasmids recovered from transformed cells, demonstrating that the

151 level of interference observed was due to the activity of Cascade-Cas3 and not by

152 varying levels of escape mutations in the target. The lack of sequence heterogeneity

153 also demonstrates that NHEJ repair could not be detected, though this type of repair is

154 rare in S. cerevisiae. Our results suggest that the programmable processive DNA

155 degradation by type I-E CRISPR-Cas system can be restored in eukaryotic cells.

157 In our initial design, Cascade, Cas3, and pre-crRNA is expressed from different

158 plasmids, and this resulted in 50\% reduction in plasmid transformation rate. To

159 improve activity we developed improved systems. Co-expression of a Cas3-Cse1 
160 fusion results in a subpopulation of Cascade complexes permanently co-localized with

161 Cas 3 , which reduced transformation by $73.5 \%$, a level similar to when J3 crRNA is

162 used against phage Lambda in E. coli expressing the LeuO activator (13). We

163 hypothesize that this fusion improves the kinetics of target degradation, as the factors

164 do not need to find each other. Further, we altered the CRISPR to produce two

165 different crRNA targeting the plasmid to potentially increase activity and at the same

166 time expressed Cas3 and pre-crRNA from the same plasmid to reduce the load of

167 carrying multiple plasmids. These modifications resulted in further reduction of

168 transformation, and combination of all improvements result in about one-in-ten

169 plasmids escaping degradation.

171 Different crRNAs result in different levels of CRISPR-Cas system interference (7, 14,

172 15), so other spacer sequences may further increase efficiency of target degradation in

173 yeast. We also speculate that the Cascade and Cas3 in the absence of nuclear

174 localization signals (NLS) locate to the cytoplasm, thus allowing only a narrow

175 window of opportunity to destroy their target plasmids while they are en route to the

176 nucleus. This likely causes a reduction in target access and interference compared to

177 bacteria and archaea, which typically lack a membrane around their nucleoid. Other

178 factors that may further improve interference in S. cerevisiae are increased expression

179 of Cas protein and pre-crRNA, codon optimization, prevention of detrimental post-

180 translational modification of Cas proteins, and co-expression of E. coli host factors

181 that may assist interference.

182 The large type I-E system is more difficult to establish heterologously than the more

183 compact class 2 systems. However, complete target clearance by type I systems is a

184 distinct advantage over inactivation of MGEs by mutagenesis using class 2 systems. 
185 To inactivate an MGE by mutation, thorough understanding of the MGEs function is 186 required to find a key component to target. Also, compared to Cas9-based systems, 187 type I systems can easily target several different sequences by adding more spacer188 repeat units to the CRISPR $(14,16)$, while current applications of Cas9 in eukaryotes 189 require insertion of multiple single guide RNA genes to achieve multiplexing (17). 190 Another benefit of our establishment of a type I-E system in S. cerevisiae is that it 191 functions as a platform for testing our current understanding of the system in a non192 native background. Such a platform would allow introduction and exclusion of e.g. 193 various proteins and other factors component to test their role in the immune system.

194 Finally, while we demonstrate interference in a eukaryote, further development could 195 reconstitute the entire adaptive capability of the system. This would require 196 expression of Cas1 and Cas2, and possibly also other factors such as IHF, indicated as 197 important for adaptation (18). As Richard Feynman stated, "what I cannot create I do 198 not understand".

\section{Materials and Methods}

201 Strains and culture media

202 All cloning work was done in E. coli strain Top10. E. coli was cultured in Lysogeny 203 Broth (LB) with aeration at $37^{\circ} \mathrm{C}$. When necessary, the media was supplemented with 204 kanamycin $(50 \mu \mathrm{g} / \mathrm{ml})$, ampicillin $(100 \mu \mathrm{g} / \mathrm{ml})$, streptomycin $(50 \mu \mathrm{g} / \mathrm{ml})$, tetracycline $205(25 \mu \mathrm{g} / \mathrm{ml})$, and chloramphenicol $(15 \mu \mathrm{g} / \mathrm{ml})$. For experiments in yeast Saccharomyces cerevisiae W303 and BY418 strains were used. S. cerevisiae BY418 have full

207 deletion of chromosomal copies of genes used for selection, unlike W303 where they 208 are inactivated by point mutations. Yeast cells were cultured in SC medium (Yeast 209 nitrogen base $0.34 \%$, ammonium sulfate $0.5 \%$, raffinose $2 \%$, quadruple SC dropout 
$210 \operatorname{mix}\left(\mathrm{CSM}\right.$, -His, -Leu, -Trp, -Ura; Formedium ${ }^{\mathrm{TM}}$, UK) at the rate of $1400 \mathrm{mg} / \mathrm{L}$ of

211 medium, pH 5.6 ). For certain induction experiments SC medium with $1 \%$ raffinose

212 was used. When necessary, the media was supplemented with adenine sulfate (80

$213 \mu \mathrm{g} / \mathrm{ml})$, uracil $(20 \mu \mathrm{g} / \mathrm{ml})$, tryptophan $(40 \mu \mathrm{g} / \mathrm{ml})$, histidine $(20 \mu \mathrm{g} / \mathrm{ml})$, and leucine $(60$

$214 \mu \mathrm{g} / \mathrm{ml}$ ). $1.5 \%$ agar was included in the medium to prepare solid medium. Yeast strains

215 were grown with aeration at $30^{\circ} \mathrm{C} .2 \%$ galactose was added to the medium for

216 induction of Gal promoter as required. For a complete list of strains used see table S1.

218 Construction of vector expressing Cascade complex

219 A Gal1-10 promoter cassette from pRS425Gal was cloned between EcoRI/BamHI

220 sites of pBlueScript II SK (+) resulting in pUDM101. All cas genes were amplified by

221 colony PCR from E. coli MG1655, see table S2 for a complete list of primers. The

222 cse 1 and $\operatorname{cse} 2$ genes were serially cloned into the pUDM101 XbaI and EcoRI sites,

223 respectively, to generate pUDM107. Similarly, cas7 and cas6e were serially cloned in

224 pRS425Gal BamHI and SalI sites, respectively, to generate pUDM108. The four

225 genes were combined by subcloning a HindIII-NotI blunt fragment from pUDM107

226 into the blunted SacI site of pUDM108, thereby generating pUDM109. The cas5e

227 gene was cloned into pRS425Gal vector to generate pUDM110. CYC1 terminator

228 cassette was PCR amplified from pYES2 and subcloned into PstI site of pBlueScript

229 II SK (+) to generate pUDM102. A CYC1-cas5e-CYC1 cassette was constructed by

230 serially cloning EcoRV-SmaI fragment from pUDM102 into the SalI and SpeI sites

231 respectively of pUDM110 to generate pUDM315. Using XhoI and NotI this cassette

232 was excised from pUDM315, blunted and sub-cloned into the NotI site of pUDM109

233 to generate the final Cascade-expressing construct, pCascade. See table S3 for

234 complete list of plasmids used in this study. 
Construction of vectors expressing Cas 3 or Cas3-Cse 1 fusion

237 The cas3 gene was PCR amplified from MG1655 to introduce flanking EcoRI sites

238 and cloned into the EcoRI site of pRS423Gal resulting in the Cas3-expressing plasmid

239 pCas3. A Cas3-Cse1 fusion fragment was amplified from pWUR657 and cloned

240 between the SpeI and NotI sites of pRS423Gal to generate pCas3::Cse1 expressing

241 the Cas3-Cse1 fusion.

\section{Construction of vectors expressing CRISPR RNA}

244 For expression of crRNA, two CRISPR cassettes, one containing 4xJ3 spacer (13)

245 PCR amplified from pWUR630 and the other containing J1-J2 spacers (a synthetic 246 array ordered from ThermoFisher Scientific Inc.) were cloned into BamHI-NotI sites

247 of pRS424Gal_Cyc1 to place CYC1 terminator directly downstream of the CRISPR

248 array. All spacers targeted the $\mathrm{J}$ gene from bacteriophage Lambda. The J1-J2

249 CRISPR, along with the flanking CYC1 terminator, was excised as a BamHI-SacI

250 fragment and cloned in pCas3 cut with the same sites to generate pCRISPR+Cas3.

251 Additionally, the same BamHI-SacI fragment was blunted and cloned in the blunted

252 SalI site of pCas3::Cse1 to generate pCRISPR+Cas3::Cse1. See table S4 for details of

253 CRISPR and targets.

254 For expression crRNA in E. coli, a minimal CRISPR array with 54 nt of the leader,

255 the J3 spacer and two repeats was cloned into pZE12Luc (19) under the control of

256 PLlacO-1. The plasmid was amplified using primers PLlacO-C and pZE-Xba and the

257 minimal CRISPR array was amplified from pWUR564 (13) using primers LA009 and

258 LA013. The array was cloned by blunt-end ligation in the leader-end so that the first 
259 position of the partial leader corresponds to transcription start of the PLlacO-1 and in

260 the other end into the XbaI-site of pZE12Luc.

\section{Construction of S. cerevisiae expressing type I-E CRISPR-Cas system}

263 The vectors constructed as described above were transformed into the desired yeast

264 strains as required. Transformation of $S$. cerevisiae was performed using lithium 265 acetate method (20).

\section{Construction of target vectors}

268 To construct pTargetHigh, the $350 \mathrm{bp}$ region of Lambda J from pWUR610 was

269 cloned into the BamHI and HindIII sites of pRS426Gal. The cloning replaced the Gal-

270 promoter fragment with the lambda DNA fragment. A low copy target vector,

271 pTargetLow, was constructed by PCR amplification of a region of Lambda $\mathbf{J}$ gene

272 using pTargetHigh as template with DR0015 and DR0016 primers and cloning the

273 fragment in the NheI site of pPS1739.

$275 \quad$ Analysis of crRNA processing

276 Sample preparation and RNA purification: Yeast

277 S. cerevisiae W303 carrying pCascade, pCRISPR (4xJ3 spacer), and pCas3 was

278 grown to stationary phase, diluted in fresh SC medium and grown with aeration at

$27932^{\circ} \mathrm{C}$ to an $\mathrm{OD}_{600}$ value of 0.3 . Galactose was then added to induce the CRISPR-Cas

280 system. $10 \mathrm{ml}$ culture was harvested by centrifugation 2,4 , and 5 hours after

281 induction. Total RNA was isolation using the hot phenol method (21). 
283 Overnight culture of E. coli BL21AI harboring pWUR397 (Cas3), pWUR400

284 (Cascade) and pLA002 (minimal J3 CRISPR array), was diluted and grown to OD 600

$285 \approx 0.3$. Cas-protein and crRNA expression was induced by addition of $0.2 \%$ arabinose 286 and $1 \mathrm{mM}$ IPTG. After $30 \mathrm{~min}, 5 \mathrm{ml}$ sample was taken and mixed with $1 \mathrm{ml}$ stop

287 solution (5\% phenol, $95 \%$ ethanol), and pelleted at $4{ }^{\circ} \mathrm{C}$. RNA was purified as

288 previously described (22).

Gel and blotting

290 Samples were run on a 10\% denaturing polyacrylamide gel (10\% polyacrylamide, 7

291 M urea, 1X Tris-borate-EDTA (TBE)) in 1X TBE. Samples were mixed with loading 292 dye (95\% deionized formamide, $0.5 \mathrm{mM}$ EDTA, $0.025 \%$ bromphenol blue/xylene 293 cyanol, and $0.025 \%$ SDS), and boiled 3 min prior to loading. The same was done 294 with the pre-labelled pUC8 size marker. $15 \mu \mathrm{g}$ of total RNA was loaded for each 295 sample. As a positive control for processed crRNA, total RNA purified from the $E$. 296 coli BL21AI carrying pWUR 397, pWUR400 and pLA002, able to prevent phage 297 infection and plasmid transformation (data not shown), was also loaded on the gel. 298 Transfer to Hybond $\mathrm{N}+$ membrane (Amersham) was done at $4{ }^{\circ} \mathrm{C}$ overnight at 200 $299 \mathrm{~mA}$. The membrane was UV crosslinked and prehydridized in Church buffer $(0.25 \mathrm{M}$ 300 sodium phosphate buffer $\mathrm{pH} 7.2,1 \mathrm{mM}$ EDTA, and 7\% SDS) for $1 \mathrm{~h}$ at $42^{\circ} \mathrm{C}$ after 301 which $0.5 \mu \mathrm{M}$ of radioactively labelled probe was added to the buffer. Hybridization 302 was done overnight. The membrane was washed two times 5 min with $2 x \mathrm{SSC}, 0.1 \%$ 303 SDS and exposed the PharosFX-system (BioRad).

304 The pUC8 size marker (Fermentas) was radioactively labeled using $\gamma^{-32} \mathrm{P}-\mathrm{ATP}$ 305 (PerkinElmer) and PNK (Fermentas) in an exchange reaction according to the 306 manufacturer's instructions. The probe, LA014, was labelled the same way in a 307 forward reaction. Excess $\gamma_{-}{ }^{32} \mathrm{P}$-ATP was removed using Illustra ProbeQuant G-50 
308 Micro column (GE Healthcare) purification according to the manufacturer's

309 instructions.

\section{CRISPR-Cas activity assays}

312 CRISPR-Cas activity was assessed by plasmid interference assays. Yeast cultures

313 grown to stationary phase and diluted in fresh SC medium containing either $1 \%$ or $2 \%$

314 raffinose as carbon source and grown with aeration at $32^{\circ} \mathrm{C}$ to an $\mathrm{OD}_{600}$ value of 0.2 ,

315 followed by addition of galactose inducer. 150-240 min after induction the cells were

316 harvested by centrifugation. The cells were then transformed with target vectors or

317 non-target control vectors using lithium acetate method (20), but excluding carrier

318 DNA. Plasmid interference by the CRISPR-Cas system was measured by plating

319 cultures transformed with target vector or non-target vector on selective media and 320 comparing TE.

\section{$\underline{\text { Surveyor assay }}$}

323 S. cerevisiae BY418 cells harboring pCascade, pCRISPR, and pCas3::Cse1, or

324 negative controls lacking pCRISPR, pCascade or both were grown in SC medium

325 with or without the galactose inducer and transformed with pTargetHigh. The

326 transformed cells were grown for 24 hours in SC medium, pelleted by centrifugation

327 and grown again in equal volume of fresh medium for 24 hours. $1.5 \mathrm{ml}$ of the culture

328 was pelleted by centrifugation and resuspended in $500 \mu \mathrm{l}$ of distilled water. The cells

329 were lysed by heating at $98^{\circ} \mathrm{C}$ for 10 minutes and $0.5 \mu \mathrm{l}$ of the lysate was used as

330 template for PCR with primers DR003 and DR004 to amplify the target region. The

331 PCR products were analyzed by electrophoresis on $2 \%$ agarose gel and used for

332 mutation detection with Surveyor mutation detection kit (IDT) as per manufacturer's 
333 instructions. The result of Surveyor assay was analyzed by Qiagen QIAxcel advanced 334 gel electrophoresis system.

\section{Acknowledgements}

337 We are deeply grateful to Erik Johansson (Umeå University), Hans Ronne (Swedish

338 University of Agricultural Sciences), Gerhart Wagner (Uppsala University), Pernilla

339 Bjerling (Uppsala University) and their respective research groups for providing

340 assistance, strains, and plasmids for this project. This project was funded by the

341 Wenner-Gren Foundations, the Swedish Research Council and the Royal Swedish

342 Academy of Sciences.

344 Author Contributions

345 ML conceived the concept. DR, GB, and ML performed experimental design and DR,

346 LA, and GB performed the experiments. DR and ML analyzed the data and wrote the 347 article.

\section{Conflict of Interest}

350 The authors declare no conflict of interest. 


\section{References}

353 1. Barrangou R, Fremaux C, Deveau H, Richards M, Boyaval P, Moineau S,

354 Romero DA, Horvath P. 2007. CRISPR provides acquired resistance

355 against viruses in prokaryotes. Science 315:1709-12.

356 2. Rath D, Amlinger L, Rath A, Lundgren M. 2015. The CRISPR-Cas immune system: Biology, mechanisms and applications. Biochimie

3. Koonin EV, Makarova KS, Zhang F. 2017. Diversity, classification and evolution of CRISPR-Cas systems. Curr Opin Microbiol 37:67-78.

4. Makarova KS, Wolf YI, Alkhnbashi OS, Costa F, Shah SA, Saunders SJ, Barrangou R, Brouns SJ, Charpentier E, Haft DH, Horvath P, Moineau S, Mojica FJ, Terns RM, Terns MP, White MF, Yakunin AF, Garrett RA, van der Oost J, Backofen R, Koonin EV. 2015. An updated evolutionary classification of CRISPR-Cas systems. Nat Rev Microbiol 13:722-36.

5. White MK, Hu W, Khalili K. 2015. The CRISPR/Cas9 genome editing methodology as a weapon against human viruses. Discov Med 19:255-62.

6. Ebina H, Misawa N, Kanemura Y, Koyanagi Y. 2013. Harnessing the CRISPR/Cas9 system to disrupt latent HIV-1 provirus. Sci Rep 3:2510. RNAs guide antiviral defense in prokaryotes. Science 321:960-4.

8. Westra ER, van Erp PB, Kunne T, Wong SP, Staals RH, Seegers CL, Bollen S, Jore MM, Semenova E, Severinov K, de Vos WM, Dame RT, de Vries R, 
consecutive binding and degradation of negatively supercoiled invader DNA by Cascade and Cas3. Mol Cell 46:595-605.

9. Sapranauskas R, Gasiunas G, Fremaux C, Barrangou R, Horvath P, Siksnys V. 2011. The Streptococcus thermophilus CRISPR/Cas system provides immunity in Escherichia coli. Nucleic Acids Res 39:9275-82.

10. Swarts DC, Mosterd C, van Passel MW, Brouns SJ. 2012. CRISPR interference directs strand specific spacer acquisition. PLoS One 7:e35888.

11. Marraffini LA, Sontheimer EJ. 2008. CRISPR interference limits horizontal gene transfer in staphylococci by targeting DNA. Science 322:1843-5.

12. Garneau JE, Dupuis ME, Villion M, Romero DA, Barrangou R, Boyaval P, Fremaux C, Horvath P, Magadan AH, Moineau S. 2010. The CRISPR/Cas

13. Westra ER, Pul U, Heidrich N, Jore MM, Lundgren M, Stratmann T, Wurm bacterial immune system cleaves bacteriophage and plasmid DNA. Nature 468:67-71. transcription activator Leu0. Mol Microbiol 77:1380-93.

14. Rath D, Amlinger L, Hoekzema M, Devulapally PR, Lundgren M. 2015. 43:237-46. spacer sequences. Nucleic Acids Res 43:10831-47. 
401 16. Zetsche B, Heidenreich M, Mohanraju P, Fedorova I, Kneppers J,

402

403

404

405

406

407

408

409

410

411

412

413

414

415

DeGennaro EM, Winblad N, Choudhury SR, Abudayyeh 00, Gootenberg JS, Wu WY, Scott DA, Severinov K, van der Oost J, Zhang F. 2017. Multiplex gene editing by CRISPR-Cpf1 using a single crRNA array. Nat Biotechnol $35: 31-34$.

17. Jinek M, Chylinski K, Fonfara I, Hauer M, Doudna JA, Charpentier E. 2012. A programmable dual-RNA-guided DNA endonuclease in adaptive bacterial immunity. Science 337:816-21.

18. Nunez JK, Bai L, Harrington LB, Hinder TL, Doudna JA. 2016. CRISPR Immunological Memory Requires a Host Factor for Specificity. Mol Cell 62:824-33.

19. Lutz R, Bujard H. 1997. Independent and tight regulation of transcriptional units in Escherichia coli via the LacR/O, the TetR/O and AraC/I1-I2 regulatory elements. Nucleic Acids Res 25:1203-10.

20. Agatep RK, R.D.; Parchaliuk, D. L.; Woods, R. A.; Gietz, R. D. 1998. Transformation of Saccharomyces cerevisiae by the lithium acetate/single-stranded carrier DNA/polyethylene glycol protocol. Technical Tips Online 3:133-137.

21. Collart MA, Oliviero S. 2001. Preparation of yeast RNA. Curr Protoc Mol Biol Chapter 13:Unit13 12.

22. Blomberg P, Wagner EG, Nordstrom K. 1990. Control of replication of plasmid R1: the duplex between the antisense RNA, CopA, and its target, CopT, is processed specifically in vivo and in vitro by RNase III. EMBO J 9:2331-40. 
Figure 1. A-D: The different variants of the type I-E CRISPR-Cas system used for expression in S. cerevisiae.

$$
\text { A }
$$

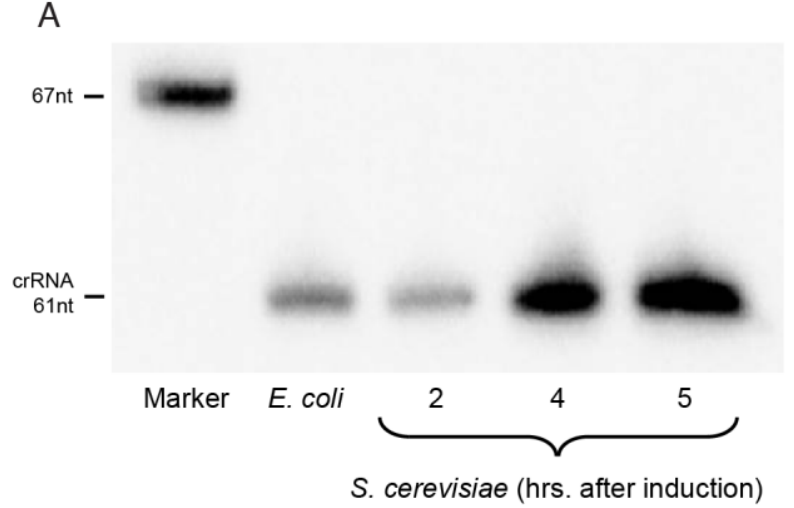

B C pCas3::Cse1
pCascade
pCRISPR D $\begin{gathered}\text { pCascade } \\ \text { pCRISPR-Cas3 }\end{gathered}$ E

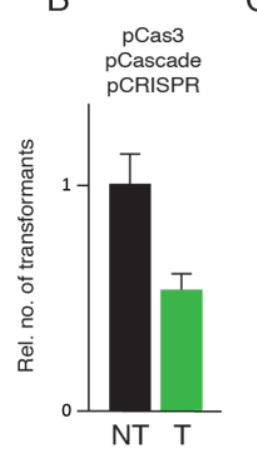

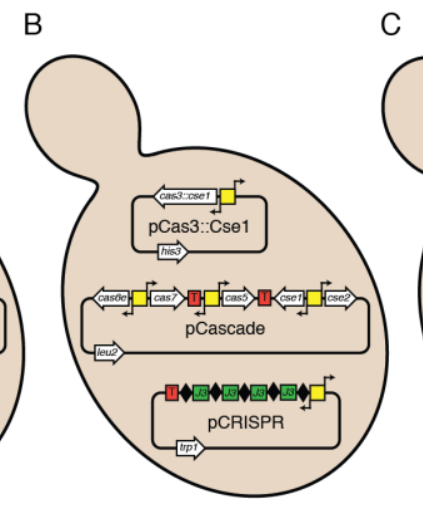
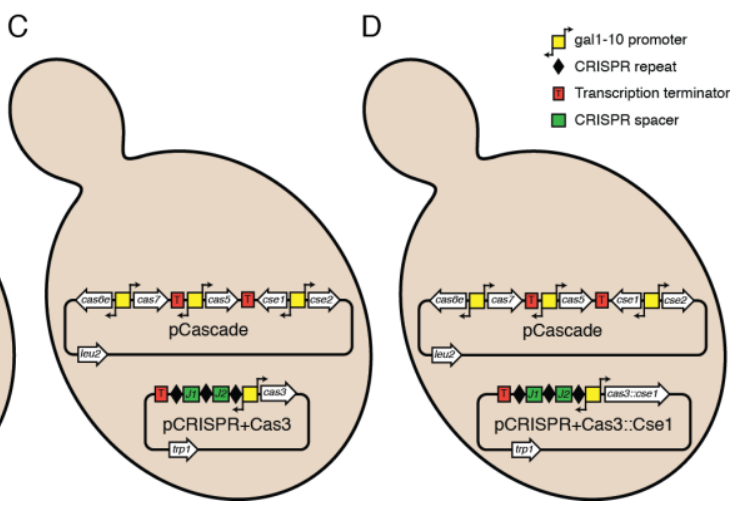

.


436 Analysis of CRISPR-Cas interference on plasmids in different S. cerevisiae strains

437 (A-B: W303, C-D: BY418) with indicated CRISPR-Cas expression system.

438 Transformation efficiency was measured for plasmids containing a target sequence

439 (T) and non-targeted parent plasmids (NT). Data in B-E is an average of three

440 independent biological replicates normalized so that relative level of transformation

441 by the non-target plasmid is equal in the different panels. Error bars indicate one 442 standard deviation. 